Document downloaded from:

http://hdl.handle.net/10251/145199

This paper must be cited as:

Ageeva, A.; Khramtsova, E.; Magin, I.; Richkov, D.; Purtov, P.; Miranda Alonso, MA.; Leshina, T. (12-0). Spin Selectivity in Chiral Linked Systems. Chemistry - A European Journal. 24(15):3882-3892. https://doi.org/10.1002/chem.201705863

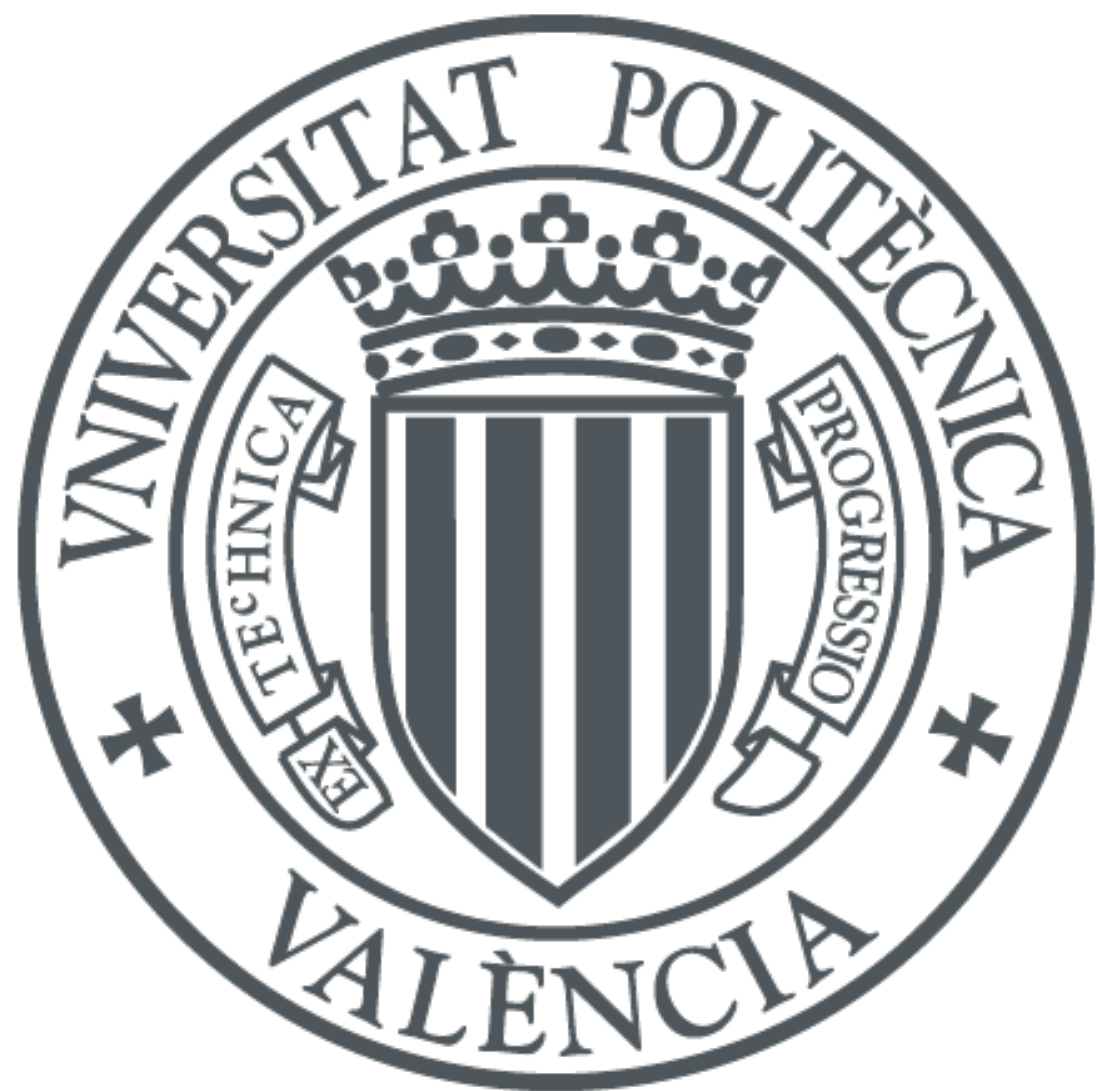

The final publication is available at

https://doi.org/10.1002/chem.201705863

Copyright John Wiley \& Sons

Additional Information

"This is the peer reviewed version of the following article: Ageeva, Aleksandra A., Ekaterina A. Khramtsova, llya M. Magin, Denis A. Rychkov, Peter A. Purtov, Miguel A. Miranda, and Tatyana V. Leshina. 2018. "Spin Selectivity in Chiral Linked Systems." Chemistry - A European Journal 24 (15). Wiley: 3882-92. doi:10.1002/chem.201705863, which has been published in final form at https://doi.org/10.1002/chem.201705863. This article may be used for non-commercial purposes in accordance with Wiley Terms and Conditions for SelfArchiving." 


\section{CHEMISTRY A European Journal}

\section{Accepted Article}

Title: Spin Selectivity in Chiral Linked Systems

Authors: Aleksandra Andreevna Ageeva, Ekaterina Khramtsova, llya Magin, Denis Rychkov, Peter Purtov, Miguel Miranda, and Tatyana Leshina

This manuscript has been accepted after peer review and appears as an Accepted Article online prior to editing, proofing, and formal publication of the final Version of Record (VoR). This work is currently citable by using the Digital Object Identifier (DOI) given below. The VoR will be published online in Early View as soon as possible and may be different to this Accepted Article as a result of editing. Readers should obtain the VoR from the journal website shown below when it is published to ensure accuracy of information. The authors are responsible for the content of this Accepted Article.

To be cited as: Chem. Eur. J. 10.1002/chem.201705863

Link to VoR: http://dx.doi.org/10.1002/chem.201705863 


\title{
Spin Selectivity in Chiral Linked Systems
}

\author{
Aleksandra A. Ageeva*[a], Ekaterina A. Khramtsova ${ }^{[a, b]}$, Ilya M. Magin ${ }^{[a]}$, Denis A. Rychkov ${ }^{[b, c]}$, Peter A. \\ Purtov $^{[a, b]}$, Miguel A. Miranda ${ }^{[d]}$, Tatyana V. Leshina ${ }^{[a]}$
}

Abstract: This work has shown spin selectivity in electron transfer $(E T)$ of diastereomers of $(R, S)$-naproxen-(S)- $N$-methylpyrrolidine and $(R, S)$-naproxen-(S)-tryptophan dyads. Photoinduced ET in these dyads is interesting due to still unexplained phenomenon of stereoselectivity in drug activity of enantiomers. The chemically induced dynamic nuclear polarization (CIDNP) enhancement coefficients of $(R, S)$-diastereomers twice exceeds those for the $(S, S)$-analogue. These facts are also interesting because spin effects are among the most sensitive even to small changes in spin and molecular dynamics of paramagnetic particles. So, CIDNP has to reflect the difference in magneto-resonance parameters (hyperfine interaction constants, $\mathrm{HFI}, \mathrm{g}$-factor difference) and lifetimes of $(R, S)$ and $(S, S)$-diastereomers paramagnetic forms. The difference in HFI values for diastereomers has been confirmed by the comparison of CIDNP experimental enhancement coefficients with calculated ones. Besides, the dependence of CIDNP enhancement coefficients ratio on diastereomers concentration ratio has been observed for naproxen- $N$-methylpyrrolidine dyad. It has been explained by the participation in ET of homo- $(R, S-R, S$ or $S, S-S, S)$ and hetero-( $R, S-$ $S, S)$ dimers of dyads. In this case, the effectivity of ET, and consequently CIDNP, is supposed to be different for $(R, S)$ - and $(S, S)$-homodimers, heterodimers and monomers. The possibility of dyads dimers formation has been demonstrated using X-Ray and NMR high-resolution techniques.

\section{Introduction}

Due to extensive application of chiral compounds, difference in enantiomer's reactivity is the focus of interest for various researchers. For example, in pharmacology this difference undoubtedly plays a significant role because chiral isomers often differ sharply in the medical properties. ${ }^{[1]}$ The subject of this study - drug naproxen (NPX) is a good example, because only one enantiomer has pharmacological activity. The molecular and cellular levels of enantiomers activity were intensively studied by their interaction with receptors and

[a] A.A. Ageeva, Dr. E.A. Khramtsova, Dr. I.M. Magin, Prof. P.A. Purtov, Prof. T.V. Leshina Laboratory of Magnetic Phenomena

Voevodsky Institute of Chemical Kinetics and Combustion SB RAS Institutskaya 3, 630090 Novosibirsk (Russia)

E-mail: al.ageeva@gmail.com

[b] Dr. E.A. Khramtsova, Dr. D. A. Rychkov, Prof. P.A. Purtov Novosibirsk State University

Pirogova 2, 630090 Novosibirsk (Russia)

[c] Dr. D. A. Rychkov

Institute of Solid State Chemistry and Mechanochemistry SB RAS Kutateladze 18, 630128 Novosibirsk (Russia)

[d] Prof. M.A. Miranda

Departamento de Química/Instituto de Tecnología Química UPVCSIC, Universitat Politècnica de València, Camino de Vera $\mathrm{s} / \mathrm{n}$, 46022 Valencia (Spain)

Supporting information for this article is given via a link at the end of the document. enzymes using biochemical methods. ${ }^{[2,3]}$ Despite the impressive results of these works, the physico-chemical nature of the difference between enantiomer's activities has been unknown yet. A possible approach for understanding this difference can be the study of isomers properties in linked systems diastereomers. This is reasonable because in living systems a chiral drug always interacts with other chiral amino acid residues located in enzyme or receptor active sites. So far as two chiral particles interact, the physical causes for difference in isomers reactivity are manifested. The most frequently discussed reason of this difference is various steric hindrances. ${ }^{[4]}$ It is also considered that drug-receptor or drug-enzyme binding can involve ET between drugs and amino acid residues.

In this regard donor-acceptor linked systems with two chiral centers (diastereomers) are frequently used as a model of the donor-acceptor interaction of chiral drugs with enzymes and receptors. ${ }^{[5-8]}$ Recently, ET in several naproxen-based dyads with two chiral centers were studied to clarify the reasons of stereoselectivity by using photochemistry and spin chemistry (CIDNP and magnetic field effect) methods. ${ }^{[6,7]}$

Methods of spin chemistry deal with radical reactions, in which the velocity and also the direction, depend on electronnuclear interactions and g-factors difference in a pair of paramagnetic particles (free radicals, radical-ions, biradicals) precursors of products. In such reactions the recombination of radical pairs turns out to be spin-selective. CIDNP effects are appeared as changes in the intensity and/or phase of lines in NMR spectra of the products of cage recombination and exit into solution due to the different recombination probability of $\alpha$ and $\beta$ nuclear spins. ${ }^{[9]}$ Since NMR technique has high precision in determining the structure of diamagnetic molecules, the analysis of CIDNP effects allows one to establish reliably the structures of paramagnetic particles - precursors of polarized molecules.

It should be emphasized that CIDNP has proven itself as one of the most informative techniques for the study ET in abovementioned dyads. The CIDNP analysis has shown that under the UV-irradiation of these dyads a reversible intramolecular ET followed by biradical-zwitterion (BZ) formation is occurred. ${ }^{[6]}$ In these systems the correlation between fluorescence quantum yields and CIDNP effectivity of diastereomers was also observed. The first mention of the differences in the chemical polarization of diastereomers for a few dyads was made by comparison of their CIDNP dependences on the solvents dielectric constants. ${ }^{[6]}$ In the work $^{[6]}$ the theoretical calculation of diastereomers CIDNP effectivity depending on the dielectric constant was also made. However, this calculation was carried out without accounts for a possible difference between magneto-resonance parameters of diastereomers BZ, where CIDNP effects are formed.

On the other hand, the EPR studies of paramagnetic forms of individual optical isomers and racemates in solid ${ }^{[8,10]}$ and solutions ${ }^{[11-14]}$ have shown the difference between the values of hyperfine interaction constants (HFI), g-factors and electron exchange interaction energy $(J)$. In these works individual chiral isomers and racemate of stable nitroxyl radicals and biradicals 
<smiles>COc1ccc2cc([C@@H](C)C(=O)N[C@H]3CCC(C(=O)OC[C@@H]4CCCN4C)C3)ccc2c1</smiles><smiles>COC(=O)[C@H](Cc1c[nH]c2ccccc12)NC(=O)[C@H](C)c1ccc2cc(OC)ccc2c1</smiles>

Figure 1. Structural formulae of dyads 1 (top) and $\mathbf{2}$ (bottom).

were studied. ${ }^{[8,10-14]}$

In this work we made an effort to consider the various causes of spin selectivity ${ }^{[\ddagger]}$ in the photoinduced ET of $(R, S)$ - and $(S, S)$-diastereomers of two naproxen based dyads (Figure 1 ). The differences of the experimental CIDNP enhancement coefficients were compared with those calculated in the framework of the model where HFI constants, g-factors difference and lifetimes of diastereomers paramagnetic forms were varied. ${ }^{[15,16]}$

In addition to the academic interest in various manifestations of chirality phenomenon, demonstration of the difference between magneto-resonance characteristics of paramagnetic forms of chiral dyads can also have practical significance. The difference in spin densities of the BZs is most likely to indicate at the difference in the electron density of diastereomers that has direct relevance to the reactivity. For example, it may be related to drug-receptors and drug-enzymes binding involving charge transfer processes.

In this work, the difference between the CIDNP enhancement coefficients was found to depend on the ratio of diastereomers concentration. It was observed upon UVirradiation of dyad's 1 diastereomers mixture.

It should be noted that for processes involving chiral systems the dependence on concentration is known to be quite typical and is usually explained by the tendency of chiral compounds to form associates. ${ }^{[17-22]}$ There is the reference data about well-known capability of naphthalene and its substituted derivatives as well as naproxen to form dimers and excimers in solid state and solutions. ${ }^{[23-27]}$ Particularly, "naproxen-naproxen" dyad excimer is described in work. ${ }^{[25]}$ For naphthalene and its derivatives dimerization occurs due to the interaction of aromatic rings. In case of naproxen, the association is believed to occur via hydrogen bonds between carboxylic groups of two molecules that are observed in solid state and exist even in diluted solutions. ${ }^{[26]}$ Literature also describes heterodimers (cocrystals) of naproxen proved to be formed with different organic

\footnotetext{
[†] Designation "spin selectivity" is also used in spintronics to describe the spin selective electron transport through the chiral medium ( $R$. Naaman, D. Waldeck, J. Phys. Chem. Lett. 2012, 3, 2178-2187).
}

compounds and biomolecules: nicotinamide, isonicotinamide, picolinamide, alanine, tyrosine, tryptophan etc. X-Ray and solidstate NMR studies have shown that these associates are formed using hydrogen bonds of naproxen carboxylic group with $\mathrm{NH}$ and $\mathrm{OH}$ groups of partners. ${ }^{[27]}$

Therefore, in this work X-Ray and NMR methods are used to confirm the possibility of dyads dimer formation. Besides, the ratios of CIDNP enhancement coefficients for diastereomers observed in solution with different ratio of diastereomers concentrations were calculated, taking into account the assumption that ET occurs in dyad dimers.

\section{Results and Discussion}

\section{Photo CIDNP Effects in diastereomers of dyads 1 and 2}

Comparison of NMR and CIDNP spectra, detected in the back intramolecular ET of dyad's $\mathbf{1}$ and $\mathbf{2}$ diastereomers demonstrates some differences between $(R, S)$ - and $(S, S)$ optical configurations. In the case of dyad 1 diastereomers there is the difference in CIDNP enhancement coefficients of methyl protons of the $\mathrm{N}$-methylpyrrolidine fragment: for $(R, S)$ configuration, it is approximately twice as high as that for $(S, S)$ (Figure 2).

On the other hand, NMR and CIDNP spectra of dyad's 2 diastereomers show significant difference in the aromatic part of NMR spectra (Figure 3). So, CIDNP spectra of $(R, S)$ configuration demonstrate the greater polarization for protons at 8' (4') and 5' positions of NPX ring (the signal of polarized protons at 5' position is just missing in the CIDNP spectrum of

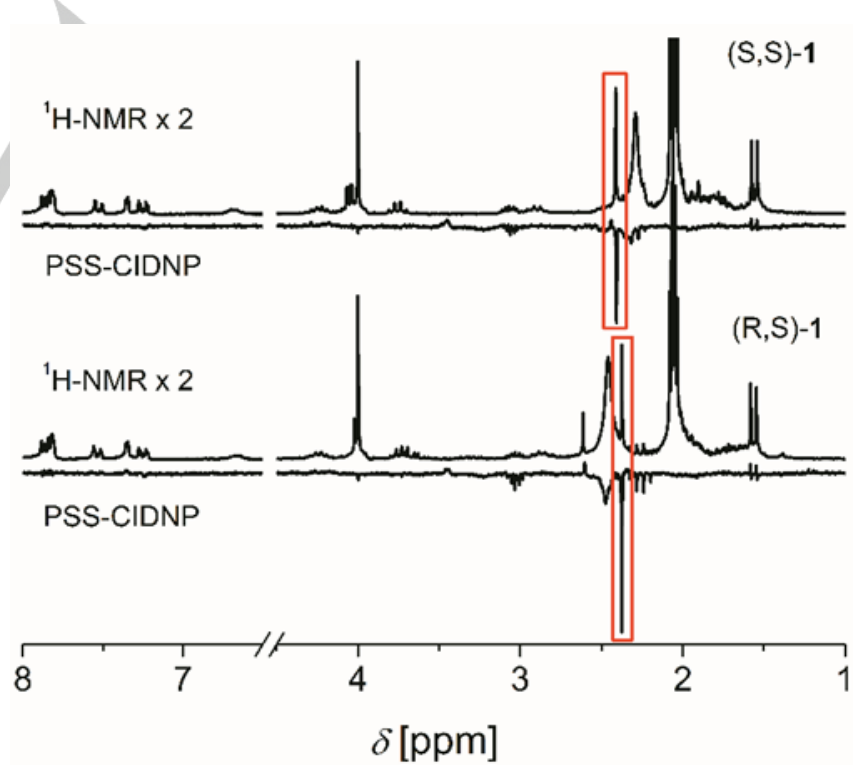

Figure 2. NMR and CIDNP spectra of dyad 1 diastereomers $(5 \mathrm{mM})$ solution in $\mathrm{CD}_{3} \mathrm{CN}\left(\mathrm{H}_{2} \mathrm{O} 0.05 \%\right)$ : $(\mathrm{S}, \mathrm{S})$ - top, $(R, S)$ - bottom. Negative polarized line situated at $2.3 \mathrm{ppm}$ (in red squared) refers to methyl protons of $\mathrm{N}$ methylpyrrolidine fragment of dyad's diastereomers. The assignment of all lines is given in the Supporting Information (Figure S1). 


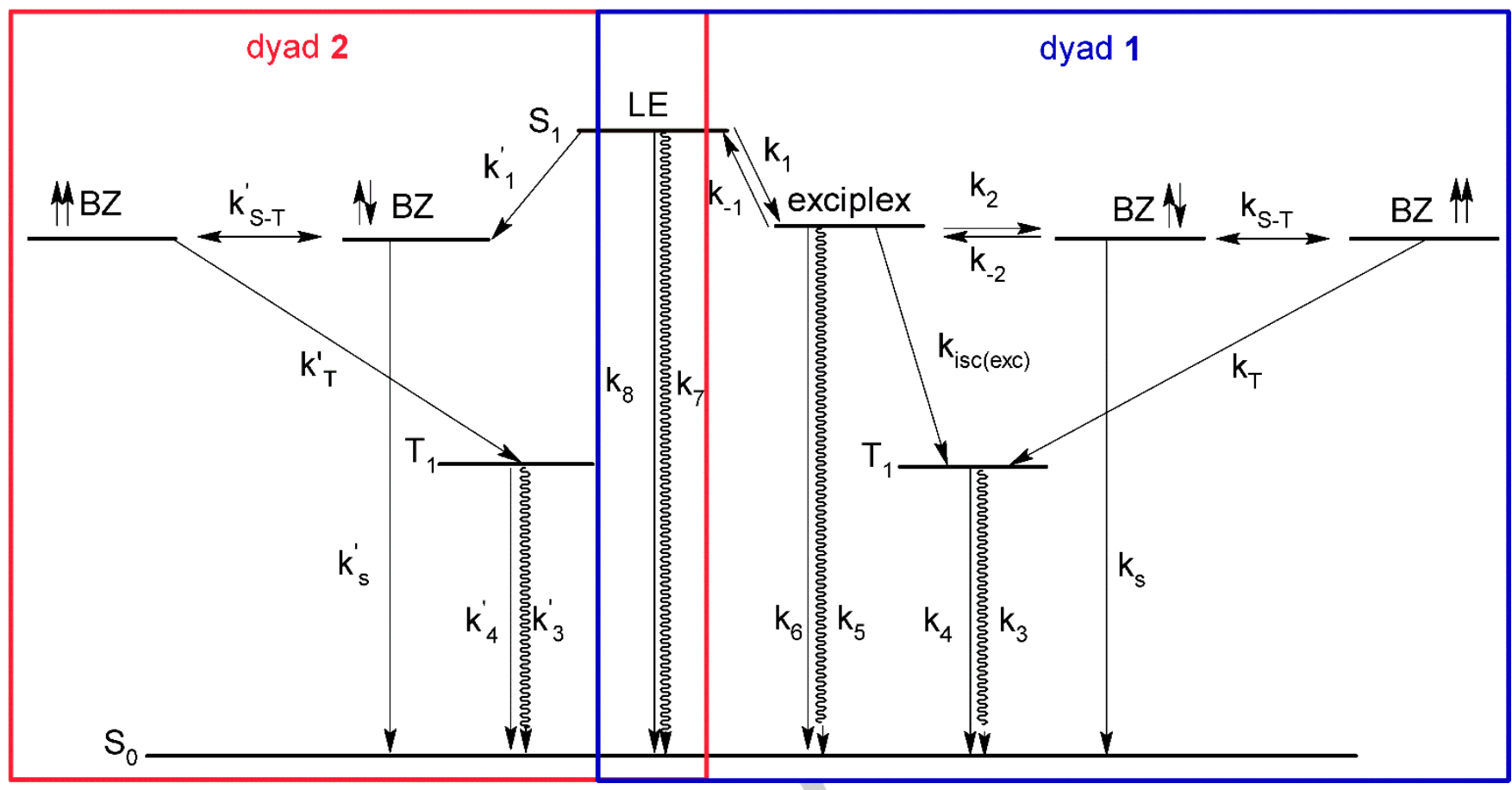

Scheme 1. Mechanism of quenching of naproxen local excited state (LE) of dyads 2 (left pathway in red rectangle) and 1 (right pathway in blue rectangle). Left pathway: $k_{1}{ }^{\prime}-\mathrm{ET}$ rate constant; $k_{s-T^{\prime}}$ - spin conversion constant in BZ; $k s^{\prime}$ and $k_{T^{\prime}}$ - rate constants of back ET from singlet and triplet spin state of BZ, respectively; $k_{3}$ ' and $k_{4}$ ' - rate constants of triplet non-radiative and radiative decay. Right pathway: $k_{1}$ and $k_{-1}$ rate constants of transformation of LE into exciplex and exciplex to LE; $k_{2}-$ ET rate constant; $k_{-2}$ - rate constant from BZ to exciplex back transfer; $k_{S-T}-$ spin conversion constant in BZ; $k_{S}$ and $k_{T}-$ rate constants of back ET from singlet and triplet spin state of BZ, respectively; $k_{3}$ and $k_{4}$ - rate constants of triplet non-radiative and radiative decay; $k_{6}$ and $k_{8}-$ fluorescence

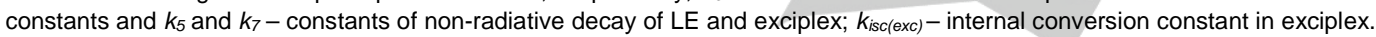

$(\mathrm{S}, \mathrm{S})$-diastereomer). Besides, there is also difference in NMR and CIDNP spectra of $\mathrm{CH}_{2}$ protons at 8 position of Trp fragment (Figure 3).

For $\mathrm{CH}_{2}$ protons the difference might be associated with not only smaller CIDNP coefficients of $(S, S)$-diastereomer but also with greater contribution from the secondary process of dyad 2 decay, involving a neutral biradical formation. This process was described in the work. ${ }^{[7]}$ In this case the chemical polarization of $\mathrm{CH}_{2}$ protons of Trp fragment might be partially masked by polarized products of dyad's decay.

The procedure of enhancement coefficients $(\boldsymbol{K})$ determination is given in the Experimental Section. The CIDNP enhancement coefficient has turned out to be appreciably higher for $(R, S)$-configuration. So, for $(R, S)$ - and (S,S)-optical configuration of dyad 1 the ratio of enhancement coefficient $K$ is $2.3 \pm 0.1$ (Figure 2).

It should be noted that for dyad 1 CIDNP effects of diastereomers were compared only in acetonitrile. As for dyad $\mathbf{2}$, same effects in several solvent mixtures were studied. The maximum value of $\boldsymbol{K}$ for diastereomers of this dyads is about two (detailed description is given in the next section).

Applying of different approaches to study CIDNP enhancement coefficients of these dyads is associated with the different mechanism of NPX excited state quenching by $N$ methylpyrrolidine and tryptophan (Scheme 1). It was shown earlier that the fast equilibrium between exciplex and BZ plays a significant role in the CIDNP formation of dyad $1^{[6]}$ This equilibrium is maximally shifted towards the BZ in polar solvent acetonitrile. In this case, one can expect that the contribution to CIDNP from processes, where exciplex is involved, will be

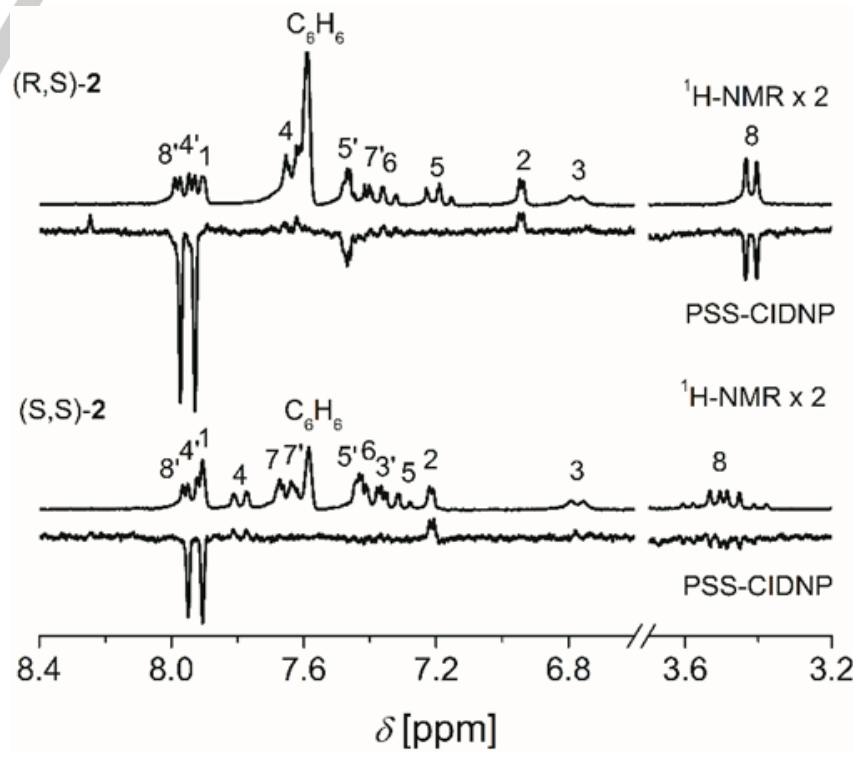

Figure 3. NMR and PSS CIDNP spectra of solutions ( $40 \% \mathrm{C}_{6} \mathrm{D}_{6}, 0.17 \% \mathrm{H}_{2} \mathrm{O}$, the rest is $\left.\mathrm{CD}_{3} \mathrm{CN}\right)$ of dyad's 2 diastereomers $(5 \mathrm{mM})$ : $(R, S)$-top, $(S, S)$-bottom The assignment of all lines is given in Supporting Information (Figures S2-S3). 
negligible. Then CIDNP values of dyad 1 protons in acetonitrile would be determined by magneto-resonance parameters of BZs. Further, in order to understand whether the enhancement coefficients of diastereomers difference is really caused by difference in the properties of BZs (magneto-resonance parameters, lifetimes), experimental and calculated ratios of CIDNP enhancement coefficients on the example of dyad 1 $(R, S)$ - and $(S, S)$-diastereomers will be compared.

As it was outlined in Introduction, CIDNP effects of dyad 1 diastereomers have been already calculated. ${ }^{[6]}$ It was a numerical modelling of the CIDNP dependence on solvent dielectric constants provided according to the Scheme $1 .{ }^{[6,7]}$ The recombination probability of radicals with $\alpha$ and $\beta$ nuclear spins was calculated by solving the basic spin chemistry equation using the Liouville equation. It was assumed that the relative motion of BZ's radical centers occurs in the Coulomb field. ${ }^{[6,28]}$ Variable parameters in the calculation were the diffusion coefficients, reflecting the change of mutual arrangement of radical centers in $\mathrm{BZ}$, and the thickness of the reaction layer, associated with the Onsager radius. BZ's lifetime was assumed to be equal to $-10^{-7} \mathrm{sec}$. for both diastereomers.

Herewith the area of the greatest difference between experimental and calculated dependencies was in the region of high dielectric constants. The cause of this discrepancy may be the inclusion of the Coulomb interaction into the calculation. Indeed, charged particles in polar media are known to act as neutral. ${ }^{[9]}$

To describe CIDNP in the solvents with high dielectric constants in this work we will use another model without taking into account the Coulomb interaction but with variation of magneto-resonance parameters and lifetimes of BZs.

In this report for theoretical modelling of CIDNP effects of dyads diastereomers, we used well-known Green function method. ${ }^{[15]}$ This approach is widely used for modelling magnetic and spin effects in radical reactions and can be applied for CIDNP effects simulation in biradicals. ${ }^{[15,16]}$ In this method recombination probability or CIDNP effects are calculated by convolution of separately computed the spin and molecular dynamics in BZ's radical centers.

In this work a pair spatial movement is described by a quite simple two-position model. ${ }^{[15,16]}$ The system may be located in or outside the reaction zone. Overall system lifetime is $\tau_{c}$, total time of system being in the reaction zone is $\tau_{r}$. The initial spin state of the radical pair is singlet, a reactive state can be singlet or triplet. To determine recombination probability one needs to find $\tau_{s}$ - total time that the system spends in the reaction zone being in a reactive singlet state. Detailed description of the approach and the modification according to the reaction scheme is presented in Supporting Information. Recombination in the scheme is defined as back electron transfer in BZ. According to abovementioned reasons concerning peculiarities of NPX excited state quenching in high polar media, we used a simplified scheme for CIDNP calculation that does not take into account the equilibrium between exciplex and BZ. In this case a set of constants is equated to zero: $k_{1}$ and $k_{-1}$ rate constants of transformation of LE into exciplex and exciplex to LE; $k_{2}$ and $k_{-2}$ - rate constant of exciplex transfer into
$\mathrm{BZ}$ and BZ back transfer into exciplex; $k_{\text {inc }}$ (exc) - internal conversion in exciplex. It should be emphasized that variation of these rate constants influences only on the scale of the effect.

In this calculation the rate constants of recombination from singlet and triplet BZ spin states in acetonitrile were defined as close in values. ${ }^{[6]}$ The calculation of CIDNP magnitude dependence on HFI constant value under various values of $\Delta g$ and characteristic time $\left(\tau_{c}\right)$ was carried out. Further we will try to trace what variations of abovementioned parameters should give the required adjustment - two-fold change in the diastereomers CIDNP effects.

It is known that the dependence of CIDNP enhancement coefficient on HFI constant value is bell-shaped for long-lived radical-ion pairs, to which $\mathrm{BZ}$ is definitely related. ${ }^{[9]}$ Location of dependence maximum is defined by $\Delta g$ and HFI constant ratio $\left(a \sim 2 \Delta g H_{0}\right)$. In the studied systems we used the following BZ magneto-resonance parameters. Polarized protons of naproxen fragment in dyad 2, according to the reference data have $a=0.5$

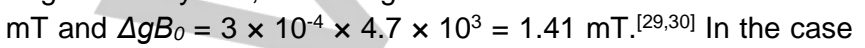
of dyad $1 \mathrm{HFI}$ constant of methyl protons of $\mathrm{N}$-methylpyrrolidine is equal to $2.9 \mathrm{mT}$, then effective constant ${ }^{[9]}$ for three protons will

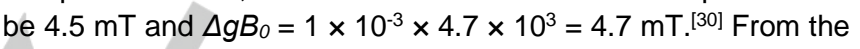
comparison of listed parameters, taking into account the bellshaped CIDNP dependence on HFI constant (Figures 4-5), one can make the following conclusion. Since both dyads have $2 \Delta g B_{0}$ values larger than $\mathrm{HFI}$ constants values, then greater polarization for $(R, S)$-isomer protons will correspond to larger $\mathrm{HFI}$ constant for this optical configuration. However, quantitative changes in $\mathrm{HFI}$ constant can be estimated only for dyad 1 . In this case HFI constants of methyl protons of $N$-methylpyrrolidine fragment are prevalent in BZ and therefore one nuclear approximation can be applied for calculation..$^{[9]}$ In case of dyad $\mathbf{2}$, $\mathrm{HFI}$ constant of aromatic protons is not prevail in BZ and shape of dependence could be much different from those in Figures 45 due to the involving in S-T evolution of other nuclei spins. ${ }^{[9]}$

If we set the lifetime of $\tau_{c}=10 \mathrm{~ns}$, close to the

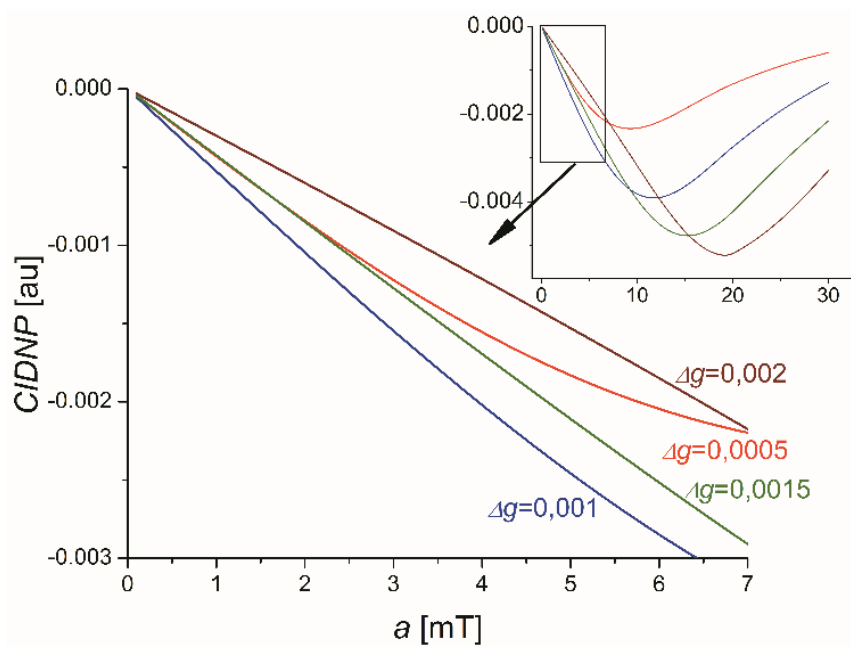

Figure 4. Initial part of CIDNP intensity dependence on HFI constant values under various parameters of $\Delta g . B=4.7 \mathrm{~T}, \tau_{c}=10 \mathrm{~ns}, \tau_{r}=1 \mathrm{~ns}$. Full curves are presented in inset. 


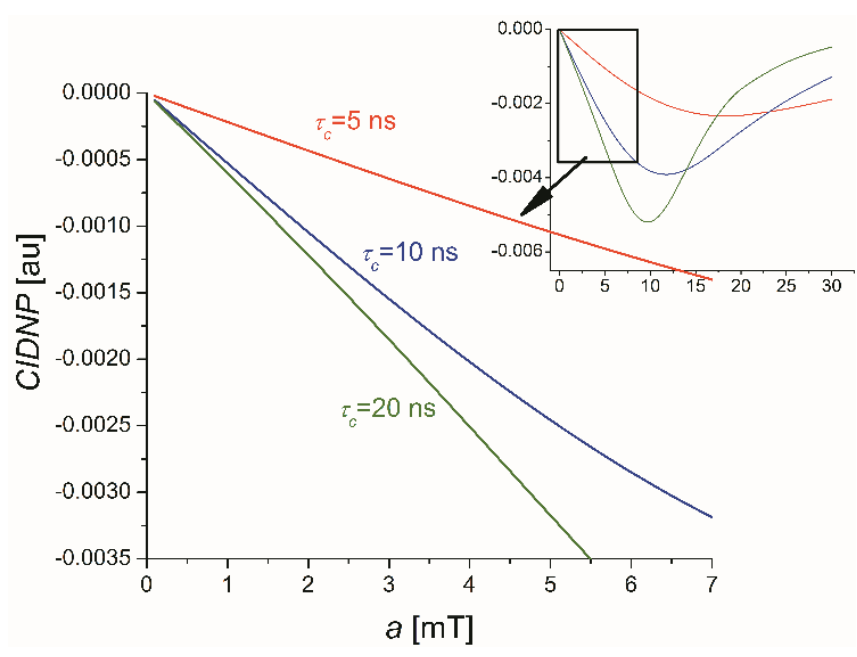

Figure 5. Initial part of CIDNP intensity dependence on HFI constant values under various parameters of $\tau_{c} . \mathrm{B}=4.7 \mathrm{~T}, T_{r}=1 \mathrm{~ns}, \Delta g=0.001$. Full curves are presented in inset.

experimental values: 7 and 9 ns for $(R, S)$ - and $(S, S)$ diastereomers of dyad $\mathbf{1}^{[6]}, \tau_{r}=1$ ns and experimental value $\Delta g=$ $10^{-3}$, then it allows one to describe the difference observed in the values of CIDNP enhancement coefficients of dyad's 1 diastereomers. In this case values of $\mathrm{HFI}$ constants for $(S, S)$ and $(R, S)$-diastereomers will differ twice (Figure 4). For instance, the $\mathrm{HFI}$ values might be $2.9 \mathrm{mT}$ and $5.8 \mathrm{mT}$, or $1.5 \mathrm{mT}$ and 2.9 $\mathrm{mT}$.

As it can be seen from Figure 4, the same result will be achieved by varying $\Delta g$ values. Note that in this case the twofolder change of CIDNP magnitude corresponds to the variation of $\Delta g$ value from 0,001 to 0,002 . The changes required in values of the g-factors are unlikely to be accepted as realistic.

In addition to variation of $\mathrm{HFI}$ constants and g-factors, it is worthwhile to consider the influence of changes in characteristic lifetimes of the studied system. According to Figure 5, CIDNP magnitude can be changed also by variation of characteristic time $\tau_{c}$. For instance, the required difference in CIDNP values can be obtained if $\tau_{c}$ of $\mathrm{BZ}$ is $5 \mathrm{~ns}$ and $10-20 \mathrm{~ns}$ for $(R, S)$ - and $(S, S)$-diastereomers, respectively. However, as it was mentioned above, BZ real lifetimes of dyad $\mathbf{1}$ diastereomers in acetonitrile change much less: from 9 to $7 \mathrm{~ns}$ for $(R, S)$ - and $(S, S)$-isomers, correspondingly. ${ }^{[6]}$ Therefore, it seems to be impossible to associate two-fold difference of CIDNP enhancement coefficient for diastereomers with difference of lifetimes $\tau_{c}$.

Note, that the variation of $\tau$, the residence time in the reactive state, by keeping the value of $\tau_{c}$ constant, also leads to the changes of CIDNP magnitude. But a size of the reaction zone was varied in previous CIDNP calculations of this system ${ }^{[6]}$, and the results point that $\tau_{r}$ can't provide the required effect.

Thus, the calculations have shown that a two-fold change in the CIDNP requires approximately the same change of $\mathrm{HFI}, \tau_{c}$ or $\tau_{\text {r }}$. The reference data about EPR investigation of diastereomers paramagnetic forms of esters, acetals ${ }^{[12]}$ and radical-ions with the general formula $\mathrm{CXYZ}-\mathrm{CH}_{2}-\mathrm{NO}^{-\bullet}$, where $\mathrm{X}$,
$\mathrm{Y}, \mathrm{Z}$ - various substituents, points to the changes of $\mathrm{HFI}$ constants in one and a half and two. ${ }^{[31]}$ Along with that, the changes in g-factors of radicals and biradicals with chiral centers do not exceed several units in the fourth decimal point. ${ }^{[32]}$ Therefore, it seems reasonable to associate the changes in CIDNP enhancement coefficient of dyad 1 diastereomers with the variation of the $\mathrm{HFI}$ constants.

\section{Dependence of $K$ on the solvent effect and ratios of diastereomers concentrations}

The impact of solvent on CIDNP coefficients of diastereomers was studied on the example of dyad 2, known not to form exciplex upon the UV-irradiation (Scheme 1). It is interesting that for dyad 2 the minimal ratio of CIDNP enhancement coefficients of diastereomers is observed in acetonitrile $-1.4 \pm 0.1$. Presence of benzene in the mixture seems to provide a greater difference between CIDNP effects of diastereomers, and this difference grows with increasing water concentration in solvent mixture (volume fraction \% CD3CN / \% $\left.\mathrm{C}_{6} \mathrm{D}_{6} / \% \mathrm{H}_{2} \mathrm{O}\right): 1.5 \pm 0.1(19.9 / 80 / 0.1)<1.7 \pm 0.1(59.8 / 40 /$ $0.2)<2.0 \pm 0.1(79.7 / 20 / 0.3)$. The same ratios were detected for the solution with concentration of diastereomers within the range of $2 \mathrm{mM}-5 \mathrm{mM}$. Perhaps, to understand the dependence of $\boldsymbol{K}$ value on solvents, it is worth taking into account the propensity of amide fragments for self-associating in polar solvents (Figure 6, I), such as: acetonitrile, acetone; and form collision complexes with aromatic compounds (Figure 6, II and III), including benzene. ${ }^{[33,34]}$

In particular, the formation of collision complexes of the amide group and indole ring with aromatic hydrocarbons was observed in NMR spectra. ${ }^{[35]}$ Note that in the NMR spectra of solutions, in which CIDNP effects were studied, noticeable (up to half a millionth part) changes of their chemical shifts, depending on benzene concentration, were observed. 2D NMR spectra of diastereomers and the dependences of their chemical shifts on the solvents are given in Supporting Information (Figures S1-S4).

Thus, the difference of $\boldsymbol{K}$ values for dyad $\mathbf{2}$ diastereomers in the mixture of solvents, containing various volume fraction of acetonitrile and benzene, might be the result of $(R, S)$ - and $(S, S)$ diastereomers association in acetonitrile. Formation of weak collisional complexes between dyad and benzene (the specific solvation effects) molecules are also possible.
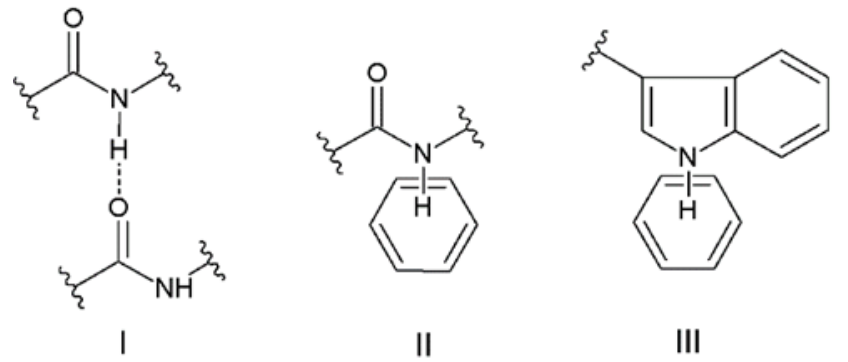

Figure 6. Self-associated (I) and collision complexes (II and III) of dyads with aromatic solvents (adapted from ${ }^{[35]}$ ). 
Because studied dyads contain "naproxen" part and "tails" - the donor groups and bridges, including amide fragment $\mathrm{C}(\mathrm{O}) \mathrm{NH}-$, they incline to form associates due to the hydrogen bonds between amide groups of two molecules (Figure $6, \mathrm{I}$ ). In fact, fraction of the dyads associates and weak collisional complexes in acetonitrile and benzene mixture may vary depending on the concentration of benzene in the mixture. Then we can assume that processes in solution would be different for $(R, S)$ - and $(S, S)$-optical configurations of the dyad 2 and might result in the $\boldsymbol{K}$ value variation in different solvents, accordingly.

Difference in the solvent effects, i.e. association of optical isomers or collisional complexes formation, can lead to a difference in the conformations of BZs. It also assumes the difference in effectivity of singlet-triplet conversion, responsible for CIDNP formation. The data indicating at the association of studied dyads, including the peculiarities of NMR spectra of diastereomers solutions, will be considered in detail below.

Apart from the measurements of CIDNP enhancement coefficients of individual diastereomers of both dyads, for dyad 1 CIDNP effects in mixtures of diastereomers with different ratio of $(R, S)$ - and $(S, S)$ - configuration in solution were measured. Observation of the polarized signals of both diastereomers under the UV-irradiation of their mixture is possible due to slight difference between chemical shifts of $\mathrm{CH}_{3}$-protons of $\mathrm{N}$ methylpyrrolidine fragments for $(R, S)$ - and $(S, S)$-configuration of dyad 1 (Figure 7). In this case, taking into account almost equal (see Experimental Section) extinction coefficients for $(R, S)$ - and $(S, S)$ - diastereomers, we believe that concentrations of the molecules, being in singlet excited state, will be proportional to the ratio of diastereomers concentrations in the solution.

Results of the measurements of observed CIDNP enhancement coefficients with different ratio of dyad 1 diastereomers are presented in Table 1 . In the first column the ratios of integral intensities for $(R, S)$ - and $(S, S)$-isomers signals

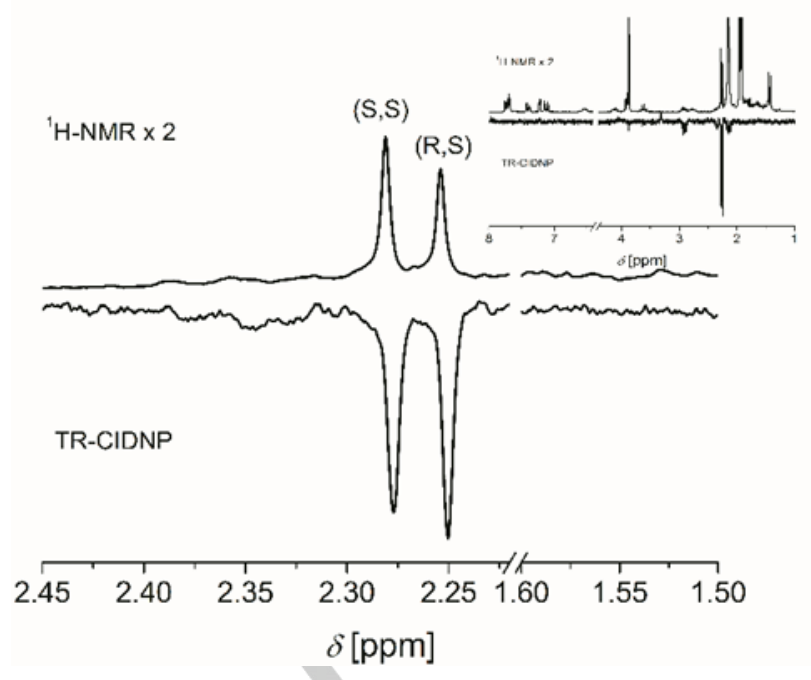

Figure 7. $\mathrm{CH}_{3}$-protons signals of $\mathrm{N}$-methylpyrrolidine fragments of dyad 1 : $(S, S)$ - (left) and $(R, S)$ - (right); in NMR and time-resolved (TR) CIDNP spectra detected for diastereomers mixture (concentration ratio $(R, S)-/(S, S)-=0.8$ in $\mathrm{CD}_{3} \mathrm{CN}\left(\mathrm{H}_{2} \mathrm{O} 0.1 \%\right)$. Full NMR and TR-CIDNP spectra are presented in insert.
Table 1. Ratios of equilibrium signals of $\mathrm{N}$-methylpyrrolidine fragment $\mathrm{CH}_{3}$ protons for $(R, S)$ - and $(S, S)-1$ dyads and ratios of CIDNP enhancement coefficients $K$ for diastereomers.

\begin{tabular}{cc}
\hline$I_{e q}^{R S}$ & $\boldsymbol{K}$ \\
\hline$I_{e a}^{S S}$ & $1.70 \pm 0.09^{[\mathrm{a}]}$ \\
0.4 & $1.80 \pm 0.09$ \\
0.7 & $1.80 \pm 0.09$ \\
0.8 & $1.9 \pm 0.1$ \\
1.0 & $2.0 \pm 0.1$ \\
1.3 & $2.3 \pm 0.1^{[\mathrm{b}]}$ \\
1.8 & $2.3 \pm 0.1$ \\
2.1 & $2.3 \pm 0.1$ \\
2.3 & \\
\hline
\end{tabular}

[a] results of the average of 3 experiments; [b] results of the average of 2 experiments

in NMR spectra before UV-irradiation are listed; in the second column $\boldsymbol{K}$ values with measurement errors are presented.

Influence of the ratio of diastereomers concentration on $\boldsymbol{K}$ value directly points to the participation of any intermolecular interactions in ET process followed by CIDNP formation. In this regard, it should be taken into account that processes of recognition and selection in chiral systems often depend on enantiomers concentration ratio. ${ }^{[17-22]}$ Also, there is the reference data that polymerization in the mixture of enantiomers is a process where a degree of chiral enrichment depends on their concentrations ratio. ${ }^{[22]}$

As it was outlined in Introduction, the dependence of processes, occurring in chiral systems, on their concentrations is often related to association of chiral compounds. In the beginning of this section, when discussing the dependence of $\boldsymbol{K}$ values of dyad $\mathbf{2}$ on the solvents, we also considered the possibility of diastereomers association via amide groups interaction. To clarify possibility of the association of dyads 2 and $\mathbf{1}$ in this section, some peculiarities of NMR spectra and XRay diffraction results will be considered.

The NMR spectra of dyad's 1 and 2 diastereomers in mixture of solvents $\mathrm{CD}_{3} \mathrm{CN} / \mathrm{C}_{6} \mathrm{D}_{6} / \mathrm{H}_{2} \mathrm{O}$ demonstrate broadening of the proton signals connected with nitrogen atoms, located in the first and third positions of dyads (Figure 8). As it can be seen, line widths of $\mathrm{NH}(1)$ and $\mathrm{NH}(3)$ protons considerably exceed that of other protons.

The protons of the amide groups of both dyads, $\mathrm{NH}(3)$, are doublets with spin-spin interaction constant equaled to $8 \mathrm{~Hz}$ and $\mathrm{NH}(1)$ protons of dyads 2 diastereomers are broad singlets.

Analysis of the lines widths of the protons located at nitrogen atoms $\mathrm{NH}(3)$ and $\mathrm{NH}(1)$ was carried out by fitting of experimental spectra with Lorentz function. The $\mathrm{NH}(3)$ lines were described as doublets, whereas in the case of the indole proton $\mathrm{NH}(1)$ the best results were obtained by fitting of one line. The results are summarized in Tables S1-S2 (see Supporting Information).

There are significant differences between line widths of doublet components of $\mathrm{NH}(3)(8 \mathrm{~Hz})$ and $\mathrm{NH}(1)$ protons $(12-13$ $\mathrm{Hz}$ ) in dyad 2 and also between $\mathrm{NH}(3)$ protons of both dyads 


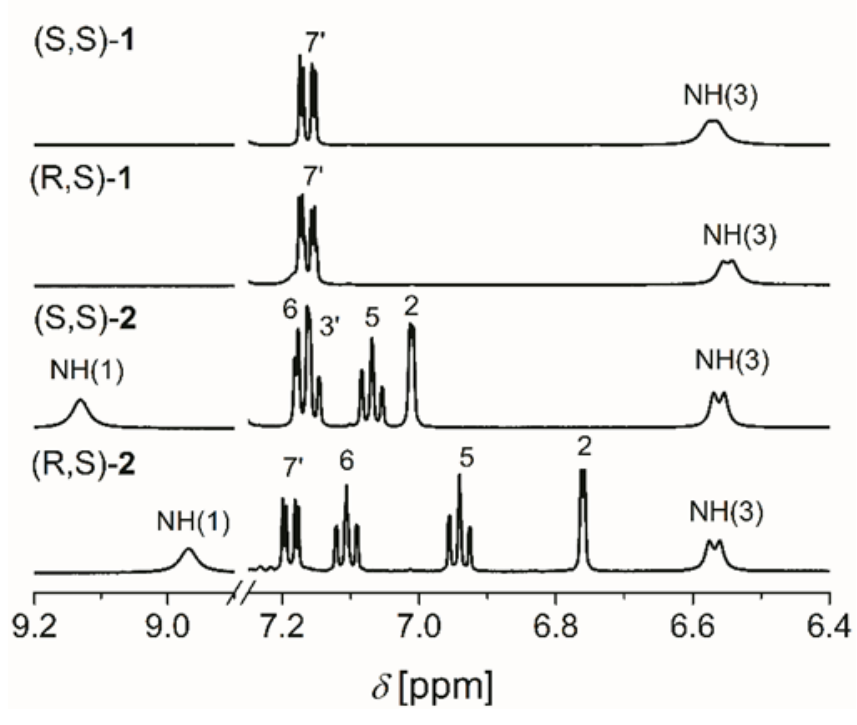

Figure 8. NMR spectra (the region of aromatic protons) of $(R, S)-/(S, S)-2$ and $(R, S)-/(S, S)-1$ dyads in the solvent mixture: $\mathrm{CD}_{3} \mathrm{CN}+0.17 \% \mathrm{H}_{2} \mathrm{O}$.

(10-12 Hz in dyad 1). These differences indicate that this broadening is not exclusively the contribution of well-known quadrupole interaction with nitrogen 14 . Then selective broadening of the amide protons of both dyads might be the result of participation of these protons in any processes of proton exchange. It may be the exchange of protons between monomers and associates of dyads or the exchange of them with water protons.

Along with that, line width of these protons of dyad 2 negligibly depends on water concentration in solutions: $W$ values changes to $1 \mathrm{~Hz}$. At the same time, this dependence for dyad's 1 diastereomers is absent at all: the values fluctuate within two hertz for both diastereomers without any connection with the concentration of water in the solution. The exchange between $\mathrm{NH}(3), \mathrm{NH}(1)$ and water protons should also lead to the dependence of the chemical shifts of these protons on water concentration in the solution. Indeed, the dependences of chemical shifts of $\mathrm{NH}(3)$ and $\mathrm{NH}(1)$ protons on water concentration were observed (Figure 9). Dependences of other protons chemical shifts on solvents mixture are presented in Supporting Information (Figure S4).

As it can be seen from Figure 9, the dependences of these protons chemical shifts on water concentration and chemical shifts of water protons itself are similar. It allows us to make a conclusion about the interaction between dyads and molecules of solvents. Since the difference in concentrations of dyad and water in solution reaches two orders of magnitude, similar changes of the water and $\mathrm{NH}$ protons chemical shifts mean that the main source of the water signal shifts is not the result of proton exchange between the water and $\mathrm{NH}$ group. Consequently, water is involved in other processes, most probably in the solvation of dyads molecules through the hydrogen bond (H-Bond) formation.

In turn, the exchange can proceed with participation of

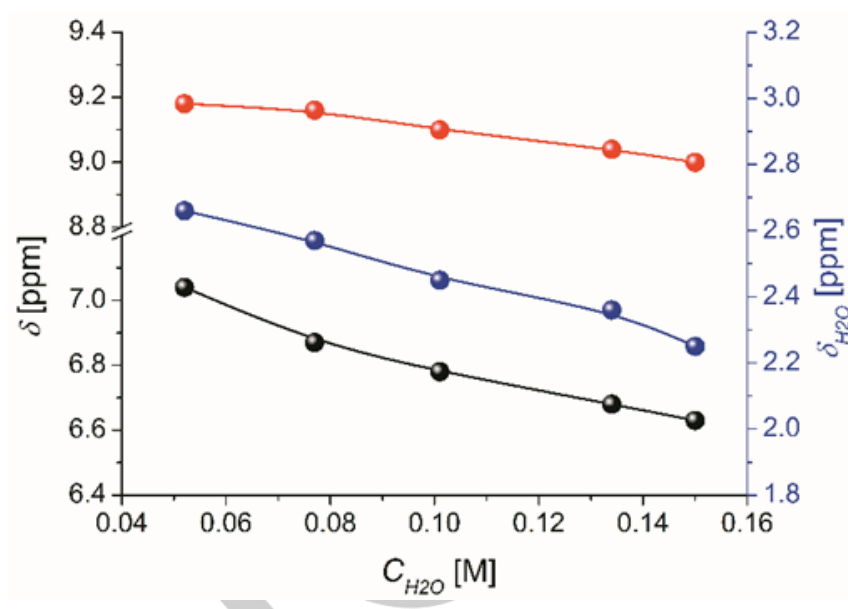

Figure 9. Chemical shift dependences of $\mathrm{NH}(1)$ (red), $\mathrm{NH}(3)$ (black) and $\mathrm{H}_{2} \mathrm{O}$ (blue) protons of $(R, S)-2$ dyad $\left(10^{-3} \mathrm{M}\right)$ on the water concentration in the mixture of $\mathrm{CD}_{3} \mathrm{CN} / \mathrm{C}_{6} \mathrm{D}_{6} / \mathrm{H}_{2} \mathrm{O}$.

associates of dyads, most likely dimers formed due to $\mathrm{H}$-bonds between the amide fragments of dyads diastereomers (Figure 6). Indeed, hydrogen bonds between the amide fragments of molecules have been proposed for dyad 2 as a result of X-Ray under low temperature data analysis. ${ }^{[36]}$ Note that X-Ray data, obtained in our work, indicates at the maintenance of these bonds at room temperature. In particular, it has been demonstrated that molecules of both $(R, S)$ - and $(S, S)$ diastereomers of dyad 2 are linked by $\mathrm{N}-\mathrm{H}$... O hydrogen bonds along axis $b$, forming similar $C^{1}{ }_{1}(5)$ and $C^{1}{ }_{1}(4)$ crystallographic motifs (Figure 10). The difference in $\mathrm{H}$-binding between two configurations of this dyad is in hydrogen acceptor group which is $-\mathrm{C}=\mathrm{O}$ fragment in ester group for $(R, S)$ - and amide group for (S,S)-2 dyad. These H-bonds differ in N...O (donor-acceptor) distance as well as in $\mathrm{N}-\mathrm{H}$... O (donor-hydrogen-acceptor) angle. For $(R, S)$-configuration $\mathrm{N}$... O distance is $2.91 \mathrm{~A}$ with $\mathrm{N}-\mathrm{H} \ldots \mathrm{O}$ angle of $167.4^{\circ}$, while for $(S, S)$-configuration N...O distance is $3.16 \mathrm{~A}$ with $\mathrm{N}-\mathrm{H} \ldots \mathrm{O}$ angle of $138.7^{\circ}$. This indicates that $\mathrm{H}$-bond in $(R, S)$-dyad is stronger than in $(S, S)$-dyad. Nevertheless, energy estimation for selected pair-wise intermolecular interactions using simple atom-atom intermolecular potentials ${ }^{[37-}$ ${ }^{38]}$ shows no significant difference for three strongest interactions in $(R, S)$ - and $(S, S)$-structures. They are $-79.3 \mathrm{~kJ} \mathrm{~mol}^{-1}(\mathrm{H}-$ bonded molecules), $-35.8 \mathrm{~kJ} \mathrm{~mol}^{-1},-28.2 \mathrm{~kJ} \mathrm{~mol}^{-1}$ for $(R, S)$ - and $81.5 \mathrm{~kJ} \mathrm{~mol}^{-1}$ (H-bonded molecules), $-31.5 \mathrm{~kJ} \mathrm{~mol}^{-1},-24.9 \mathrm{~kJ} \mathrm{~mol}^{-}$ 1 for $(S, S)$-. Thus, total energy of pair-wise interaction of molecules connected via $\mathrm{H}$-bonds are approximately equal despite the difference in $\mathrm{H}$-bonds' strength difference. This is an example of weak Van der Waals interactions optimization to pack molecules more effectively. Comparison of the results for dyad 2 diastereomers study by X-ray diffraction with NMR data also shows the possibility for amide protons - $\mathrm{NH}(3)$ to participate in the formation of intermolecular $\mathrm{H}$-bonds in solutions. Since the same behavior (selective line broadening) is characteristic for the $\mathrm{NH}$ protons of diastereomers of dyad $\mathbf{1}$, we suggest that this conclusion might be extended to the dyad 1 diastereomers. 

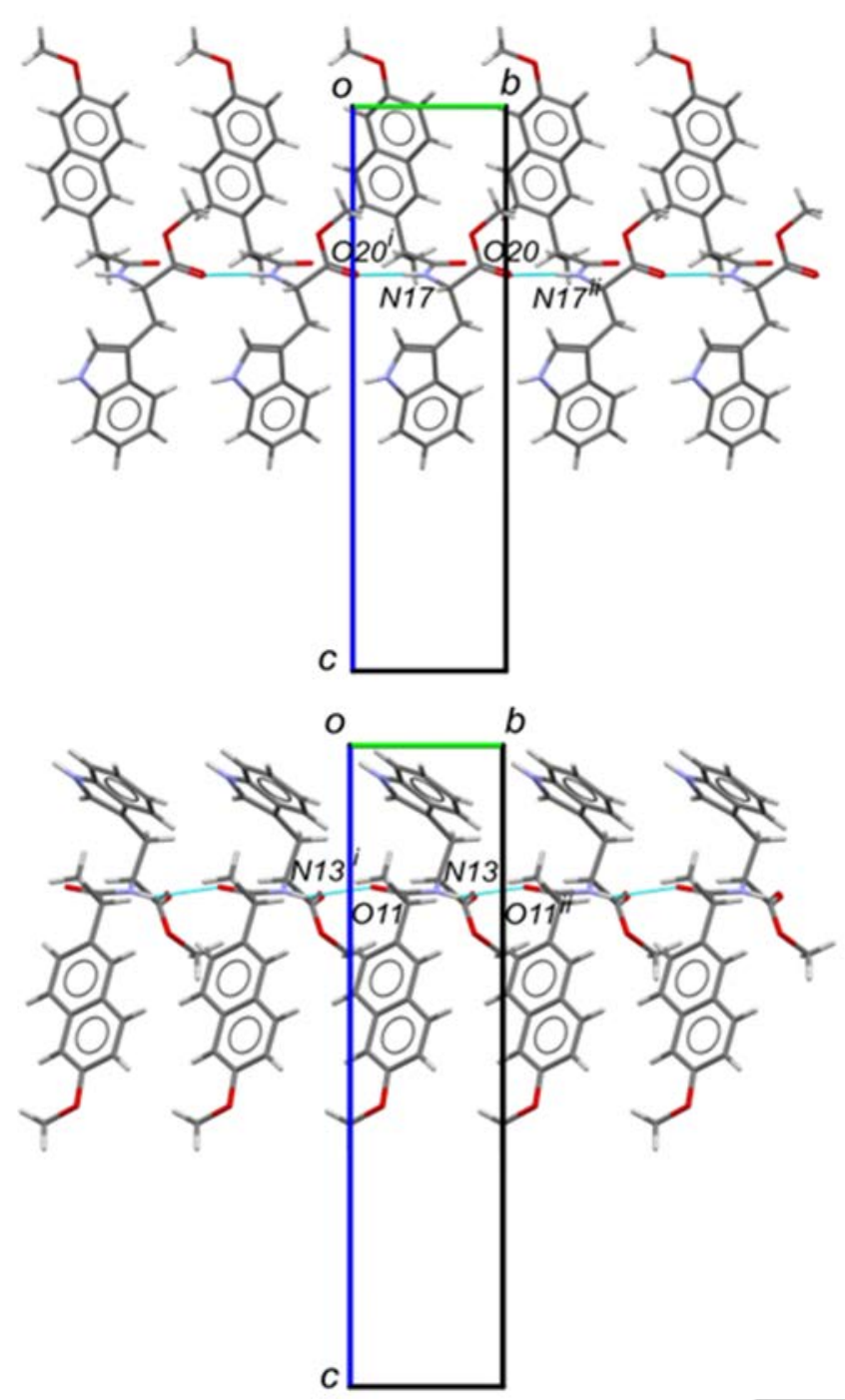

Figure 10. Structural motifs along axes b,c and a (top down) for (S,S)configuration of $\mathbf{2}$ dyad. Data obtained from X-Ray diffraction analysis for dyad's single-crystal at room temperature.

Dimerization may occur via $\mathrm{H}$-bond formation of amide group $\mathrm{NH}$ proton of one molecule with carbonyl group of other according to Scheme 2. If dimers are involved in chemical exchange, it has to be the slow exchange between positions in which the residence time of protons is greater than the inverse distance between the exchanging lines in NMR spectrum. Since the $\mathrm{NH}(3)$ protons split through the interaction with the neighboring proton $(\mathrm{J}=8 \mathrm{~Hz})$ is appeared, we can conclude that this is the case of a slow exchange with the residence time of proton $1 / J \approx 10^{-1} \mathrm{sec}$.

Due to a noticeable influence of water concentration on proton chemical shifts, association of diastereomers may depend on water concentration (Figure 9; the second equilibrium in the Scheme 2). Therefore, the analysis of the experimental data both NMR and X-Ray (for dyad 2), leads to the conclusion about possibility of the association of both dyads. It is most likely

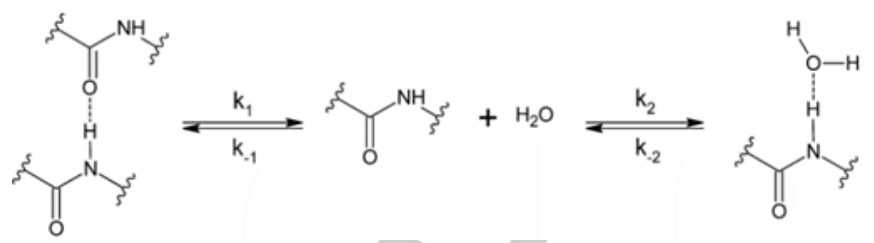

Scheme 2. Possible ways of participation of the amide fragment of studied dyads in $\mathrm{H}$-bond formation.

the dimers. The dependence of different protons chemical shifts on benzene concentration points to the formation of also weak solvation complexes with benzene.

Further, we will make an effort to imagine how the dimerization of diastereomers can change CIDNP enhancement coefficients. As it follows from the data of Table 1, the greatest $\boldsymbol{K}$ value is observed for those samples, where the concentration of $(R, S)$-isomer twice or more exceeds those of $(S, S)$-analogue. In this case, the difference is similar to that obtained in the experiments with UV-irradiation of individual optical isomers in solutions. However, at the lower isomer ratios the difference between $(R, S)$-isomer and $(S, S)$-analogue becomes smaller.

We assume that effectivity of back ET, occurring in the excited dimers, accompanied by CIDNP formation, will be different for homo- and heterodimers consisting of $(R, S)-(R, S)$ or $(S, S)-(S, S)$ and $(R, S)-(S, S)$ monomers, respectively (see Scheme 3 ). In this scheme the product of back ET is polarized dyad (RS", SS $)$, where CIDNP enhancement coefficients for homo- and heterodimers differ as $\alpha$ and $\beta$, correspondingly. We presume that $\alpha>>\beta$, i.e. homodimers formation increases ET and CIDNP effectivities.

To follow the relation between the magnitude of CIDNP and the concentration of dimers, the calculation of dimers concentrations was performed. For getting the dimers concentration, the approximation of quasi steady-state concentrations was used.

$$
\begin{aligned}
& D_{R S, R S} \stackrel{h v}{\longrightarrow}\left(R S^{*}: R S\right) \rightarrow \alpha_{R S} R S^{\#} \\
& D_{S S, S S} \stackrel{h v}{\longrightarrow}\left(S S^{*}: S S\right) \rightarrow \alpha_{S S} S S^{\#} \\
& D_{R S, S S} \stackrel{h v}{\longrightarrow}\left(R S^{*}: S S\right) \rightarrow \beta_{R S} R S^{\#} \\
& D_{R S, S S} \stackrel{h v}{\longrightarrow}\left(R S: S S^{*}\right) \rightarrow \beta_{S S} S S^{\#}
\end{aligned}
$$

Scheme 3. Photoinduced processes, occurring in homo-( $\left.D_{R S, R S}, D_{S S, S S}\right)$ and hetero- $D_{R S, S S}$ chiral dyad dimers.

Assumption was also made that the concentration of dimers is much larger than the monomers - [D $R S, R S],\left[D_{S S, S S}\right]$, $\left[D_{R S, S S}\right] \gg[R S]$, [SS]. The rate constants of dimers formation are determined by the following equilibrium. 


$$
\begin{aligned}
& R S+R S \underset{K_{R S}^{-}}{\stackrel{K_{R S}^{+}}{\rightleftharpoons}} D_{R S, R S} \\
& S S+S S \underset{K_{S S}}{\stackrel{K_{S S}^{+}}{\rightleftharpoons}} D_{S S, S S} \\
& R S+S S \underset{K_{R S, S S}}{\stackrel{K_{R S, S S}^{+}}{\rightleftharpoons}} D_{R S, S S}
\end{aligned}
$$

Conditions of quasi steady-state approximation are:

$$
\begin{gathered}
\frac{d\left[D_{R S, R S}\right]}{d t}=K_{R S}^{+}[R S]^{2}-K_{R S}^{-}\left[D_{R S, R S}\right]=0 \\
\frac{d\left[D_{S S, S S}\right]}{d t}=K_{S S}^{+}[S S]^{2}-K_{S S}^{-}\left[D_{S S, S S}\right]=0 \\
\frac{d\left[D_{R S, S S}\right]}{d t}=K_{R S, S S}^{+}[R S] \cdot[S S]-K_{R S, S S}^{-}\left[D_{R S, S S}\right]=0
\end{gathered}
$$

Then, we obtain the equations for dimers stability constants:

$$
\begin{gathered}
K_{R S}=\frac{K_{R S}^{+}}{K_{R S}^{-}}=\frac{\left[D_{R S, R S}\right]}{[R S]^{2}} \\
K_{S S}=\frac{K_{S S}^{+}}{K_{S S}^{-}}=\frac{\left[D_{S S, S S}\right]}{[S S]^{2}} \\
K_{R S, S S}=\frac{K_{R S, S S}^{+}}{K_{R S, S S}^{-}}=\frac{\left[D_{R S, S S}\right]}{[R S] \cdot[S S]}
\end{gathered}
$$

Following equations present the relation of initial diastereomers concentration [RS]o and [SS]o and current concentrations of dimers and individual diastereomers:

$$
\begin{aligned}
& {[R S]_{0}=[R S]+2\left[D_{R S, R S}\right]+\left[D_{R S, S S}\right]} \\
& {[S S]_{0}=[S S]+2\left[D_{S S, S S}\right]+\left[D_{R S, S S}\right]}
\end{aligned}
$$

According to the abovementioned approximation, the monomer concentration is sufficiently less than the dimer concentration; in latter equations the monomer member can be neglected.

$$
\begin{aligned}
& {[R S]_{0}=2\left[D_{R S, R S}\right]+\left[D_{R S, S S}\right]} \\
& {[S S]_{0}=2\left[D_{S S, S S}\right]+\left[D_{R S, S S}\right]}
\end{aligned}
$$

Hence, using (3) the following equation can be obtained:

$$
K_{R S}[R S]^{2}=\frac{1}{2}\left(2 K_{S S}[S S]^{2}+[R S]_{0}-[S S]_{0}\right)
$$

and substituting (6) to (5), after trivial transformations we can get biquadratic equation concerning [SS]

$$
\begin{aligned}
& \left(K_{R S, S S}{ }^{2} \frac{K_{S S}}{K_{R S}}-4 K_{S S}{ }^{2}\right)[S S]^{4}+ \\
& +\left(4 K_{S S}[S S]_{0}+K_{R S, S S} \frac{2[R S]_{0}-[S S]_{0}}{2}\right)[S S]^{2}-[S S]_{0}^{4} K_{R S}=0
\end{aligned}
$$

Solving this equation and choosing the physically meaning root we obtain the expression for the $(S, S)$-diastereomer's equilibrium concentration.

$$
\begin{aligned}
& {[S S]^{2}=\frac{1}{4} \frac{8 K_{S S} K_{R S}[S S]_{0}-K_{R S, S S}^{2}\left([S S]_{0}-[R S]_{0}\right)-\sqrt{D}}{K_{S S}\left(4 K_{S S} K_{R S}-K_{R S, S S}^{2}\right)}} \\
& D=K_{R S, S S}^{4}\left([S S]_{0}^{2}-2[S S]_{0}[R S]_{0}+[R S]_{0}^{2}\right)+16 K_{S S} K_{R S} K_{R S, S S}^{2}[S S]_{0}[R S]_{0}
\end{aligned}
$$

Keeping in mind (1), (3) and (5), the ratio of CIDNP magnitude (I) for $(R, S)$ - and $(S, S)$ - will be determined by the following equation:

$$
\begin{aligned}
& \left.\frac{I_{R S}}{I_{S S}}=\frac{\alpha_{R S}\left[D_{R S}, R S\right.}{\alpha_{S S}\left[D_{S S, S S}\right]+\beta_{R S}\left[D_{R S}\left[D_{R S}\right] S S\right.}\right] \\
& =\frac{\beta_{R S}[R S]_{0}+\left(\alpha_{R S}-\beta_{R S}\right)\left([R S]_{0}-[S S]_{0}+2 K_{S S}[S S]^{2}\right)}{\beta_{S S}[S S]_{0}+\left(\alpha_{R S}-\beta_{R S}\right) 2 K_{S S}[S S]^{2}}
\end{aligned}
$$

If we divide the ratio (9) to the initial diastereomers concentrations, we get the following expression for the calculated analog of CIDNP enhancement coefficient ratio $K$ (12) (see Experimental Section), $\boldsymbol{K}_{c}(\mathrm{RS} / \mathrm{SS})=I_{R S}[S S]_{0} / I_{S S}[R S]_{0}$.

According to our abovementioned assumption, if we set [DSS,SS], [DRS,RS], [D RS,SS] > [SS], [RS] and use the concentrations of $[R S]_{0}$ and $[S S]_{0}$ about $1 \mathrm{mM}$, then the values of stability constants are $K_{R S}, K_{S S}, K_{R S, S S} \sim 10^{5} \mathrm{M}^{-1}$.

Now we consider the dependence of $K_{c}$ on the $(R, S)$ diastereomer concentration $[R S]$, using the $(S, S)$-diastereomer concentration, $[S S]_{0}=10^{-3} \mathrm{M}$, and simultaneously varying the stability constants of homo- and heterodimers ( $\left.\alpha_{R S}, \alpha_{S S}, \beta_{R S}, \beta_{S S}\right)$.

Since it was found experimentally that the CIDNP enhancement coefficient for $(R, S)$-configuration twice exceeds those values for $(S, S)$-analogue, we can set $\alpha R S=2 \alpha s s$. The modeling also shows that CIDNP effectivity ratios have a weak correlation with monomers polarization coefficients $\beta$. Other results of the modeling are shown in Figures 11-12.

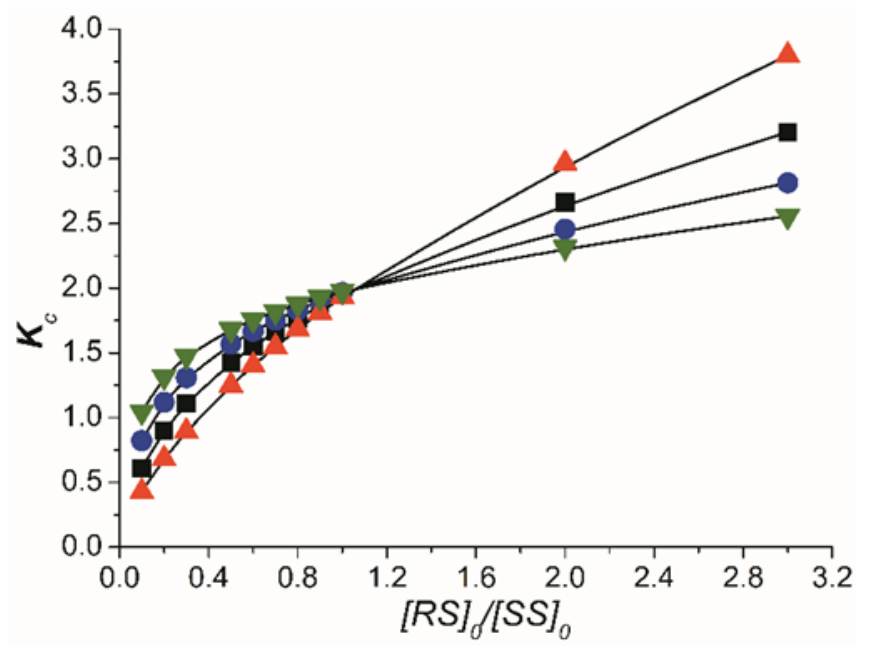

Figure 11. $\boldsymbol{K}_{\boldsymbol{c}}$ dependence on $(R, S)$-diastereomer concentration for different values of homodimer stability constants. $\left[S S_{0}\right]=10^{-3} \mathrm{M} ; K_{R S, S S}=10^{5} ; \alpha_{S S}=10$, $\alpha_{R S}=20 ; \beta_{S S}=1 ; \beta_{R S}=1 ; \boldsymbol{m}: K_{R S}=10^{5}, K_{S S}=10^{5} ; \bullet: K_{R S}=2 \cdot 10^{5}, K_{S S}=10^{5} ; \mathbf{\Delta}:$ $K_{R S}=5 \cdot 10^{4}, K_{S S}=10^{5} ; \boldsymbol{\nabla}: K_{R S}=2 \cdot 10^{5}, K_{S S}=2 \cdot 10^{5}$. 


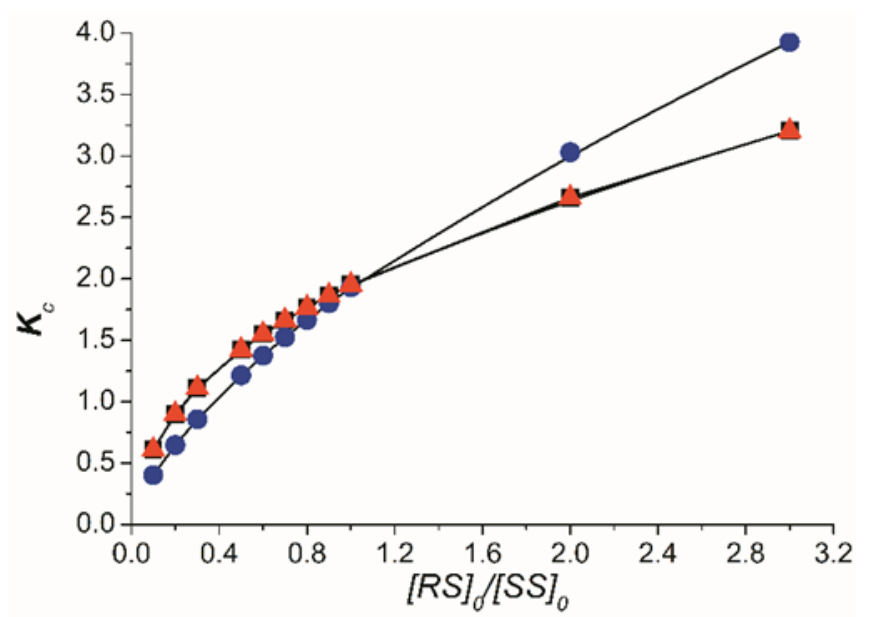

Figure 12. $\boldsymbol{K}_{\boldsymbol{c}}$ dependence on $(R, S)$-diastereomer concentration for different

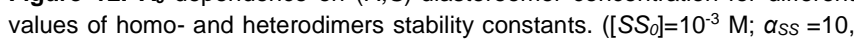
$\alpha_{R S}=20, \beta_{R S}=1, \beta_{S S}=1 ; \mathbf{m}: K_{R S}=10^{5}, K_{S S}=10^{5}, K_{R S, S S}=10^{5} ; \bullet: K_{R S}=10^{5}, K_{S S}=10^{5}$ $\left.K_{R S, S S}=1.5 \cdot 10^{5} ; \mathbf{\Delta}: K_{R S}=2 \cdot 10^{5}, K_{S S}=2 \cdot 10^{5}, K_{R S, S S}=2 \cdot 10^{5}\right)$.

The chosen model supposes that with increasing the value of $[R S]_{0} /[S S]_{0}$ ratio, the curve goes to a plateau with the level, corresponding to the value of $\alpha / \beta$ ratio. It is seen from Figure 11 that increase in values of the constants $K_{R S}, K_{S S}$ accelerates output of the curve on a plateau. Rise of cross-coupling constant value leads to the same result as decreasing of homogeneous constants values. Simultaneous increasing of all constants results in no changes in curve's shape.

As it can be seen from Figure 13, this model adequately describes the dependence of CIDNP enhancement coefficients ratio on diastereomers concentration ratio in the investigated range of diastereomers concentrations. This allows us to conclude that the effectivity of back ET and, consequently, CIDNP formation in BZs of studied dyads actually can be

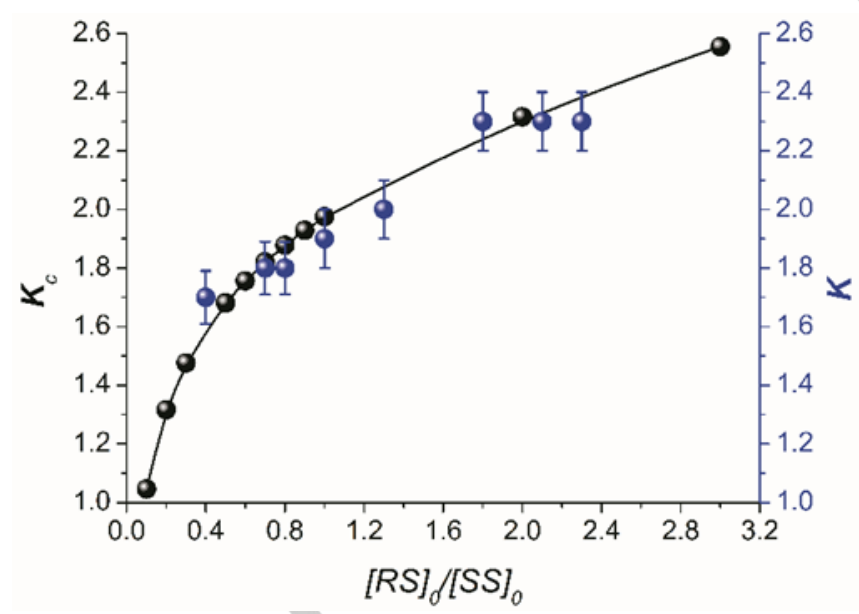

Figure 13. Experimental dependence of RS/SS diastereomers CIDNP enhancement coefficients ratio $\boldsymbol{K}$ (data from Table 1, blue dots) and calculated $\boldsymbol{K}_{\boldsymbol{c}}$ (black) dependence on $(R, S)$-diastereomer concentration, [SSo] $=10^{-3} \mathrm{M}$; $\alpha_{S S}$ $=10, \alpha_{R S}=20, \beta_{R S}=1, \beta_{S S}=1 ; K_{R S}=2 \cdot 10^{5}, K_{S S}=2 \cdot 10^{5}, K_{R S, S S}=1 \cdot 10^{5}(\circ)$. influenced by dyads self-association. It may be the appearance of so-called chiral catalysis - enrichment of solution by one optical isomer, occurring in presence of the same isomer as a catalyzer. ${ }^{[17-22]}$ In our case it is the appearance of the difference between efficiency of ET in homo- and heterodimers, concentrations of which depend on the ratio of diastereomers concentration.

Besides, it is worth noting that the above conclusion on the relation of CIDNP coefficients differences with HFI constants differences for the diastereomers should be attributed not to individual BZ, but to BZ incorporated in dimers. Therefore, an additional reason of the difference between HFI constants in BZs of diastereomers might be influence of dimerization on BZs conformation.

\section{Conclusions}

Thus, in this work spin selectivity of ET in (R)- and (S)naproxen linked with electron donors - $N$-methylpyrrolidine and tryptophan were observed. The difference in the CIDNP enhancement coefficients of $(R, S)$ - and $(S, S)$-diastereomers was shown to be a result from the difference in the HFI constants in dyads paramagnetic forms - BZs. Such short-lived particles are difficult to detect by EPR and optics methods, and in this particular case, the CIDNP analysis seems to be the most suitable technique to trace the difference between paramagnetic forms of diastereomers. Besides, we assume that the process of intramolecular ET, leading to formation of $B Z$ and creation of CIDNP, respectively, occurs with participation of the diastereomers dimers. This hypothesis allows us to explain the dependence of CIDNP enhancement coefficients ratio on diastereomers concentrations. In this case, the influence of one optical configuration on ET efficiency in the diastereomer with the same optical configuration might be an example of chiral catalysis.

\section{Experimental Section}

\section{Preparation of Solutions}

Methods of dyad's synthesis are described in previous papers. ${ }^{[6,7]}$ Stock solutions for CIDNP measurements were prepared in solvents: deuteroacetonitrile (D99.9\%) or deuterobenzene (D99.8\%) deuteroacetonitrile mixture; both solvents of Aldrich; and water (Izotop).

\section{${ }^{1} \mathrm{H}-\mathrm{NMR}$ and CIDNP Measurements}

${ }^{1} \mathrm{H}$-NMR experiments were performed using Bruker DPX $(200 \mathrm{MHz}$ $1 \mathrm{H}$ operating frequency, $\mathrm{T}(90)=3.3 \mu \mathrm{s})$ and Bruker AVHD $(500 \mathrm{MHz} 1 \mathrm{H}$ operating frequency, $\mathrm{T}(90)=11.2 \mu \mathrm{s})$ NMR spectrometers. ${ }^{1} \mathrm{H}$ pseudo steady-state $(\mathrm{PSS})^{[39]}$ and time-resolved $(\mathrm{TR})^{[40]}$ CIDNP experiments were performed on DPX-200 NMR spectrometer. The samples in standard $5 \mathrm{~mm}$ Pyrex NMR tubes were irradiated directly in the probe of the Bruker DPX 200 NMR spectrometer. A Lambda Physik EMG 101 MSC excimer laser was used as a light source $(308 \mathrm{~nm}, 100 \mathrm{~mJ}$ at output window, $20 \mathrm{~mJ}$ per pulse in sample volume, pulse duration $15 \mathrm{~ns}$ ). The samples were deoxygenized by argon bubbling for 15 minutes right 
before irradiation. Extinction coefficients at $\lambda=308 \mathrm{~nm}$ of dyad's diastereomers were measured by using an Agilent 8453 spectrophotometer (Agilent Technologies) and turned out to be almost equal: $(R, S)-2$ and $(S, S)-2 \varepsilon=850 \pm 10 \mathrm{M}-1 \mathrm{~cm}-1 ;(R, S)-1$ and $(S, S)-1$ $=840 \pm 10 \mathrm{M}-1 \mathrm{~cm}-1$.

To determine CIDNP enhancement coefficients of protons in both studied dyads the integral intensities of polarized lines and lines in NMR spectra detected before UV-irradiation were measured. All obtained values are the results of averaging at least of three measurements.

All ${ }^{1} \mathrm{H}$-NMR spectra detected before and after dyads photolysis were recorded on Bruker AVHD NMR spectrometer. Chemical shifts were measured relative to the internal standard of TMS.

Determination of CIDNP enhancement coefficients of 1 and 2 dyad's diastereomers.

For quantitative determination of the CIDNP enhancement coefficients the ratios of intensities of polarized and equilibrium signals in NMR spectra were divided by biradical-zwitterion concentrations [BZ] of studied dyads. The concentration of BZs was determined by incident energy (laser pulse $20 \mathrm{~mJ}$ at $308 \mathrm{~nm}$ ), irradiated volume of the sample equal to $25 \mathrm{~mm}^{3}$ in standard $5 \mathrm{~mm}$ Pyrex NMR tubes and a fraction of energy absorbed by the chromophore, which is quenched through ET. So the concentration of BZ was determined by the following equation:

$[B Z]=\left(1-10^{-\varepsilon l l}\right) \frac{\lambda E k_{E T} \tau_{f l}}{h c N_{A} V}$

where $\varepsilon$ - extinction coefficient of dyad measured at wavelength $308 \mathrm{~nm}$, $\mathrm{M}^{-1} \mathrm{~cm}^{-1} ; C$ - dyads concentration, $\mathrm{M} ; \mathrm{I}$ - path length of light in the sample $\mathrm{cm} ; \lambda$ - wavelength of the laser light, $\mathrm{m} ; E$ - energy of the laser pulse, $\mathrm{J}$; $k_{E T}-$ rate constant of ET, $\mathrm{s}^{-1} ; \tau \neq l$ - fluorescence lifetimes of studied dyads, $\mathrm{s} ; \mathrm{V}$ - irradiated volume fraction of the sample, I; $N_{A}$ - Avogadro's constant, $\mathrm{mol}^{-1} ; h$ - Plank constant, $\mathrm{J} \mathrm{s}$; c - speed of light, $\mathrm{m} \mathrm{s}^{-1}$.

Value of $k_{E T}$ can be calculated using the fluorescence quantum yields $\left(\varphi_{f I}\right)$ and lifetimes of dyads taken from ref. ${ }^{[6]}$ :

$k_{E T}=\frac{1-\varphi_{f l}}{\tau_{f l}}$

Thus, the CIDNP enhancement coefficient is equal to the observed CIDNP enhancement coefficient divided to the BZ concentration. Further, we will operate only with the ratio of enhancement coefficients determined by the following equation:

$K=\frac{I_{\text {pol }}^{R S} \times I_{\text {eq }}^{S S} \times[B Z]_{S S}}{I_{\text {eq }}^{R S} \times I_{\text {pol }}^{S} \times[B Z]_{R S}}$

where $I_{p o l}$ - intensity of polarized signals of the aromatic protons at the 8'position of dyad 2 naphthalene ring or $\mathrm{CH}_{3}$-protons of $\mathrm{N}$-methylpyrrolidine fragment of 1 ; $I_{\text {eq }}$ - equilibrium signal intensity of the same protons, [BZ] - concentration of biradical-zwitterion, $\mathrm{M}$.

It is worth noting that a fraction of the irradiated volume to the detected volume of the sample tubes as well as hardware gains of acquired spectrum was identical in all experiments.

\section{X-Ray Diffraction Measurement}

To study the possibility of the formation of dyad's associates $X$ Ray diffraction measurement was provided. Crystals of $(S, S)-2$ and
$(R, S)$-2 were grown using slow evaporation technique as described in ref. ${ }^{[41]}$ Crystal structures of $(S, S)-2$ (CCDC 1494730) and $(R, S)-2$ (CCDC 1494743) were solved from single crystal X-Ray diffraction at ambient conditions using an Oxford Diffraction Gemini Ultra diffractometer with a two-dimensional detector Ruby CCD (graphite monochromator, Mo Ka radiation, $\lambda=0.71073 \AA$ ). CrysAlisPro software [Agilent, CrysAlisPro, Agilent Technologies Ltd, Yarnton, England, 2014] was used for cell refinement, data collection and processing. All crystal structures were solved using OLEX2. Both dyad crystals proved to be same forms as described in ref ${ }^{[42]}$, no evidence of polymorphism ${ }^{[43]}$ was found during these experiments.

\section{Acknowledgements}

The work was supported by the Russian Foundation for Fundamental Research (14-03-00192).

Authors are very grateful to Prof. V. Plyusnin and Dr. A. Kruppa for fruitful discussion.

Keywords: spin selectivity • electron transfer • CIDNP • chirality - dimers

[1] G.-Q. Lin, Q.-D. You, J. F. Cheng, Chiral Drugs: Chemistry and Biological Action, John Wiley \& Sons Inc., New Jersey, 2011, pp. 3-29, 323-381.

[2] K. Krasulova, M. Siller, O. Holas, Z. Dvorak, P. Anzenbacher, Xenobiotica 2015, 315-324.

[3] Q. Shen, L. Wang, H. Zhou, H. Jiang, L. Yu, S. Zeng, Acta Pharm. Sinic 2013, 34, 998-1006.

[4] K. C. Duggan, D. J. Hermanson, J. Musee, J. J. Prusakiewicz, J. L. Scheib, B. D. Carter, S. Banerjee, J. A. Oates, L. J. Marnett, Nature Chem. Biol. 2011, 7, 803-809.

[5] M. C. Jiménez, U. Pischel, M. A. Miranda, J. Photochem. Photobiol. C. 2007, 8, 128-142

[6] E. A. Khramtsova, D. V. Sosnovsky, A. A. Ageeva, E. Nuin, M. L. Marin P. A. Purtov, S. S. Borisevich, S. L. Khursan, H. D. Roth, M. A. Miranda, V. F. Plyusnin, T. V. Leshina, Phys. Chem. Chem. Phys. 2016, 18 12733-12741.

[7] E. A. Khramtsova, A. A. Ageeva, A. A. Stepanov, V. F. Plyusnin, T. V Leshina, Z. Phys. Chem. 2017, 231, 609-623.

[8] S. Abad, U. Pischel, M. A. Miranda, Photochem. Photobiol. Sci. 2005, 4 69-74.

[9] K. M. Salikhov, Yu. N. Molin, R. Z. Sagdeev, A. L. Buchachenko, Spin Polarization and Magnetic Effects in Radical Reactions, Akademiai Kiado, Budapest, 1984.

[10] P. A. Levkin, H.-J. Kolb, A. I. Kokorin, V. Schurig, R. G. Kostyanovsky Chirality 2006, 18, 232-238.

[11] V. K. Khlestkin, Yu. I. Glasachev, A. I. Kokorin, R. G. Kostyanovsky, Mend. Commun. 2004, 14, 318-320.

[12] M. Maeurer, H. B. Stegmann, Chem. Ber. 1990, 123, 1679-1685.

[13] R. W. Kreilick, J. Becher, E. F. Ullman, J. Am. Chem. Soc. 1969, 91, 5121-5124.

[14] P. Schuler, F.-M. Schaber, H. Stegman, E. Jansen, Magn. Reson. Chem. 1999, 37, 805-813.

[15] A.B. Doktorov, S.A. Mikhailov, P.A. Purtov. Chem. Phys. 1992, 160, 223237.

[16] I. M. Magin, P. A. Purtov, A. I. Kruppa, T. V. Leshina, J. Phys. Chem. A 2005, 109, 7396-7401. 
[17] P. Yin, Z.-M. Zhang, H. Lv, T. Li, F. Haso, L. Hu, B. Zhang, J. Bacsa, Y. Wei, Y. Gao, Y. Hou, Y.-G. Li, C. L. Hill, E.-B. Wang, T. Liu, Nat. Commun. 2015, 6, 1-8.

[18] K. Soai, T. Kawasaki, A. Matsumoto, Chem. Rec. 2014, 14, 70-83.

[19] F. Frank, Biochim. Biophys. Acta 1953, 11, 459-463.

[20] R. Hegstrom, D. Kondepudi, Chem. Phys. Lett. 1996, 253, 322-326.

[21] Y. Ishida, T. Aida, J. Am. Chem. Soc. 2002, 124, $14017-14019$

[22] K. Sato, Y. Itoh, T. Aida, Chem. Science 2014, 5, 136-140.

[23] N. O. Dubinets, A. A. Safonov, A. A. Bagaturyants, J. Phys. Chem. A 2016, 120, 2779-2782.

[24] J. B. Aladekomo, J. B. Birks, Proc. R. Soc. Lond. A 1965, 284, 551-565.

[25] M. C. Jim'enez, U. Pischel, M. A. Miranda, J. Photochem. Photobiol. C 2007, 8, 128-142.

[26] M. Pracz, M. Franska, R. Franski, J. Chem. Pharm. Res. 2012, 4, 231238.

[27] H. Kerr, R. Softley, K. Suresh, P. Hodgkinson, I. Evans, Acta Crystallogr C Struct Chem. 2017, 73, 168-175.

[28] A. B. Doktorov P. A. Purtov, Chem. Phys. Rep., Russian edition 1985, 4, 1587-1594.

[29] J. Bernstein, Polymorphism in Molecular Crystals, vol. 14, Oxford University Press, New York, 2002.

[30] N. N. Saprygina, O. B. Morozova, N. P. Gritsan, O. S. Fedorova, A. V. Yurkovskaya, Russ. Chem. Bull. Int. Ed. 2011, 60, 2529-2587.

[31] W. Schwarz, K.-M. Dangel, G. Jones, J. Bargon, J. Am. Chem. Soc. 1982, 104, 5686-5689.

[32] B. Gilbert, J. Larkin, R. Norman, J. Chem. Soc., Perkin Trans. 2 1972, 9, $1272-1279$.

[33] M. Maurer, W. Hiller, B. Muller, H. Stegmann, Chem. Ber. 1992, 125, $857-865$.

[34] L. Pejov, Chem. Phys. Lett. 2001, 339, 269 -278.

[35] M. Muñoz, R. Ferrero, C. Carmona, M. Balón, Spectrochem. Acta 2004, 60, $196-200$.

[36] J. Hatton, R. Richards, Mol. Phys. 1961, 5:2,139-152.

[37] I. Vayá, I. Andreu, M. C. Jiménez, M. A. Miranda, Photochem. Photobiol. Sci. 2014, 13, 224-230.

[38] A. Gavezzotti, Acc. Chem. Res. 1994, 27, 309-314.

[39] A. Gavezzotti, G. Filippini, J. Phys. Chem. 1994, 98, 4831-4837.

[40] G. L. Closs, R. J. Miller, J. Am. Chem. Soc. 1979, 101, 1639-1641.

[41] M. Goez, Chem. Phys. Lett. 1992, 188, 451-456.

[42] D. A. Rychkov, S. G. Arkhipov, E. V. Boldyreva, J. Appl. Crystallogr. 2014, 47, 1435-1442.

[43] I. Vayá, I. Andreu, M. C. Jiménez, M. A. Miranda, Photochem. Photobiol. Sci. 2014, 13, 224-230.
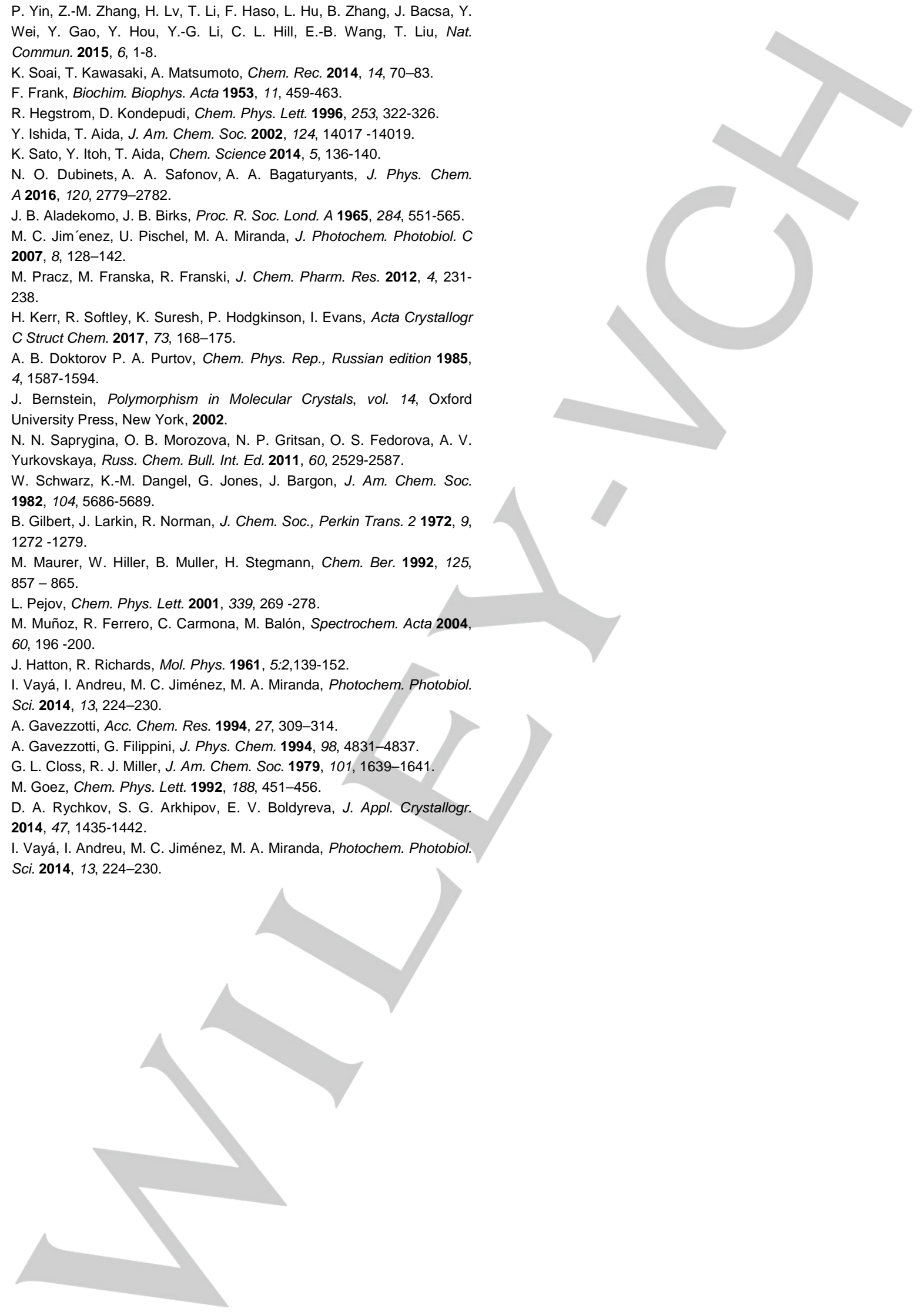


\section{FULL PAPER}

A new manifestation of chirality was observed - the difference in the chemically induced dynamic nuclear polarization (CIDNP) enhancement coefficients for diastereomers of donor-acceptor dyads. The difference in the CIDNP effects, formed in electron transfer (ET), is interpreted as a result of the difference in HFI constants in the paramagnetic forms of diastereomers. The ET is supposed to occur in dimers formed by dyads in solution.

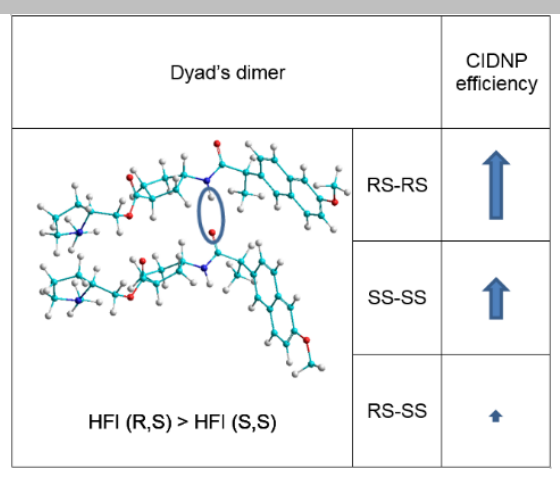

Aleksandra A. Ageeva*, Ekaterina A. Khramtsova, Ilya M. Magin, Denis A. Rychkov, Peter A. Purtov, Miguel A. Miranda, Tatyana V. Leshina

Page No. - Page No.

Spin Selectivity in Chiral Linked Systems 